\title{
Mediastinite no Hospital de Base do Distrito Federal: incidência em seis anos
}

\author{
Luiz Carlos SCHIMIN*, Raelson de Lima BATISTA*, Frederico Carlos Cordeiro de MENDONÇA*
}

RBCCV 44205-584

Schimin LC, Batista RL, Mendonça FCC - Mediastinite no Hospital de Base do Distrito Federal: incidência em seis anos. Rev Bras Cir Cardiovasc 2002; 17(2): 36-39.

RESUMO: Realizada análise dos prontuários de pacientes adultos submetidos à operação cardíaca com esternotomia no Hospital de Base do Distrito Federal no período de seis anos. Determinou-se incidência de mediastinite em 15 (1,09\%) pacientes, dentre 1388 operações cardíacas consecutivas com esternotomia, realizadas no período de janeiro de 1995 e março de 2001. Diversas variáveis foram consideradas, como sexo, idade, tipo de operação, tempo de permanência em respirador, tempo de permanência em Unidade de Terapia Intensiva, uso de antibióticos e germes isolados em culturas de ferida operatória.

DESCRITORES: Mediastinite, cirurgia. Procedimentos cirúrgicos cardíacos, efeitos adversos. Esterno, cirurgia. Osteomielite, esterno.

\section{INTRODUÇÃO}

A mediastinite e a osteomielite esternal estão entre as complicações mais sérias de uma esternotomia (1-5). Excluindo-se as operações que ocorrem após um trauma torácico, estima-se que a mediastinite ocorra em aproximadamente $2 \%$ (com variação de 0,5 a $5 \%$ ) dos pacientes que são submetidos à esternotomia mediana ${ }^{(1,2)}$. Tornou-se, portanto, uma complicação infreqüente, porém perigosa em função dos altos índices de morbi-mortalidade envolvidos $(1,2,6,7)$.

A maioria dos casos apresenta-se dentro de duas semanas após a incisão esternal ${ }^{(1,2)}$. Aspectos diagnósticos importantes dos pacientes que desenvolvem mediastinite precocemente, logo após operação cardíaca, incluem febre persistente acima de $38^{\circ} \mathrm{C}$ após - $4^{\circ}$ dia de pós-operatório, uma condição tóxica sistêmica, leucocitose, bacteremia e secreção purulenta pela ferida cirúrgica ${ }^{(1)}$. Eritema de ferida, dor ou instabilidade esternal anormal e alargamento de mediastino podem estar ausentes, ou clinicamente inaparentes, no início do desenvolvimento da mediastinite ${ }^{(1,8)}$.

O diagnóstico da doença requer um alto índice de suspeita e uma pesquisa vigorosa, repetitiva, por evi- dências de drenagem purulenta através da ferida esternal, em pacientes que estão persistentemente febris dentro da 1aㅗ semana após a operação, nos quais não foi identificado nenhum outro foco óbvio de infecção ${ }^{(5,6,9,10)}$.

Vários exames complementares podem ser utilizados para o diagnóstico da mediastinite, destacandose a tomografia computadorizada de tórax, que se mostra particularmente útil quando há envolvimento do esterno (osteomielite), havendo destruição óssea parcial ou total. A presença isolada de 'ar' em nível mediastinal, como referência para o diagnóstico por imagem de processo infeccioso em mediastino, perde seu valor no pós-operatório imediato em decorrência da manipulação cirúrgica mediastinal prévia ${ }^{(1,2,7-111)}$.

Existem vários fatores de risco pré, intra e pósoperatórios para o desenvolvimento da infecção ${ }^{(3-5,10)}$. Os elementos pré-operatórios dizem respeito, na maioria das vezes, às condições inerentes ao paciente, como idade avançada, diabetes, doença pulmonar obstrutiva crônica, doenças sistêmicas do colágeno etc. Já os fatores intra e pós-operatórios são na sua grande parte controláveis, pois envolvem o tempo de circulação extracorpórea, número de artérias mamárias internas (direita e esquerda) como

Trabalho realizado no Hospital de Base do Distrito Federal. Brasília, DF.

Recebido para publicação em dezembro de 2001.

* Do Hospital de Base do Distrito Federal (HBDF).

Endereço para correspondência: Luiz Carlos Schimin - Unidade de Cirurgia Cardíaca - Hospital de Base do Distrito Federal - SHIS 102, Asa Sul, Brasília, Distrito Federal, Brasil. CEP: 70000-000. Tel: (61)325-4410. e-mail: abramer@terra.com.br 
enxertos vasculares na revascularização do miocárdio; quantidade de sangramento pós-operatório requerendo múltiplas transfusões sangüíneas e/ou reoperações; necessidade de suporte inotrópico prolongado, bem como uso de ventilação mecânica demorada, dentre outros.

O espectro de microrganismos que provocam mediastinite inclui Stafiloccocus (aureus e epidermidis) em aproximadamente $50 \%$ dos casos e bacilos gram negativos em $40 \%$ dos pacientes, respeitando-se as variações inerentes a cada Serviço cirúrgico, mapeadas pelo perfil bacteriano de cada hospital ${ }^{(1,12)}$.

Embora os métodos terapêuticos propostos para a abordagem da mediastinite sejam muitos e controversos, a maioria dos estudos tem apontado para a necessidade de tratamento cirúrgico com debridamento amplo de tecidos necróticos, refechamento esternal e instalação de sistemas de irrigação com soluções antibióticas e/ou anti-sépticas ${ }^{(1-4,8)}$. Novos métodos de tratamento têm sido reportados, mais recentemente envolvendo abordagens menos agressivas do ponto de vista cirúrgico, como a utilização de toracoscopia para realização do debridamento, associada ou não ao uso de câmara hiperbárica de oxigênio (6,9-11).

Indiscutivelmente, a utilização de antibióticos de largo espectro e, preferencialmente, escolhidos a partir de testes de sensibilidade antimicrobianas, é consenso, os quais deverão ser mantidos por longo período de tempo ${ }^{(1)}$.

A mortalidade associada à infecção varia muito, e parece estar relacionada com o retardo na introdução do tratamento; pacientes diagnosticados e tratados agressivamente dentro de um mês da operação têm mortalidade de aproximadamente $10 \%$, enquanto que aqueles tratados mais tardiamente têm mortalidade de aproximadamente $25 \%(1,8,9,11)$.

O presente artigo tem como objetivo relatar a incidência de mediastinite no Serviço de Cirurgia Cardíaca do Hospital de Base do Distrito Federal, no período de seis anos.

\section{CASUÍSTICA E MÉTODOS}

Foram estudados, retrospectivamente, os prontuários dos pacientes que desenvolveram mediastinite entre 1388 (um mil trezentas e oitenta e oito) operações cardíacas consecutivas com esternotomia realizadas no Hospital de Base do Distrito Federal, no período compreendido entre $1^{\circ}$ de janeiro de 1995 e 31 de março de 2001 (seis anos e dois meses).

As variáveis estudadas foram armazenadas em programa de computador EPI-INFO 6.04b do CDC Atlanta/EUA, a partir do qual foram realizados cálculos estatísticos com análise e interpretação.

\section{RESULTADOS}

Ocorreram $15(1,09 \%)$ casos de mediastinite após operação cardíaca com esternotomia. Destes, 6 (40\%) pacientes foram a óbito (Gráfico 1). A operação de revascularização do miocárdio esteve relacionada com a ocorrência de $73,3 \%$ dos casos de mediastinite (11 pacientes), enquanto que o restante ocorreu após operação de Bentall-De Bono (2 casos), troca valvar mitral (1 paciente) e após pericardiectomia (1 caso) (Gráfico 2).

\section{GRÁFICO1}

FREQÜÊNCIA DE ÓBITO

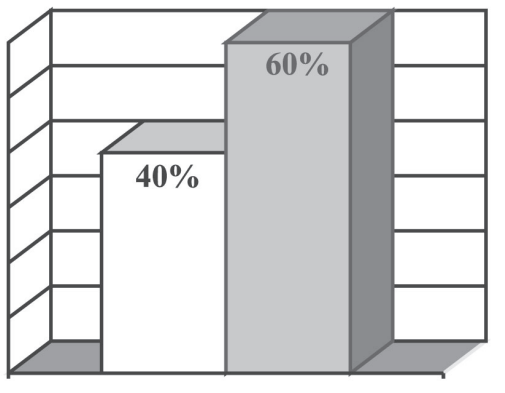

$\square$ Óbitos

$\square$ Recuperados

GRÁFICO2

PREVALÊNCIA DE OPERAÇÕES

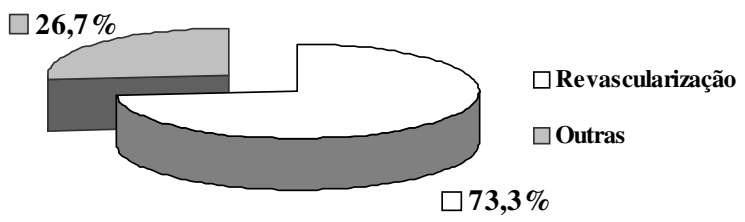

A doença foi diagnosticada antes da alta hospitalar do paciente na grande maioria dos casos (93\%), sendo que 14 ocorrências de mediastinite foram registradas após a primeira semana de pós-operatório.

Quanto ao sexo, a grande maioria dos pacientes era do sexo masculino $(86,7 \%)$, enquanto que apenas $2(13,3 \%)$ casos ocorreram em mulheres (Gráfico 3). A idade média foi de 54,8 anos (38 a 70 anos).

GRÁFICO 3

DISTRIBUIÇÃO POR SEXO

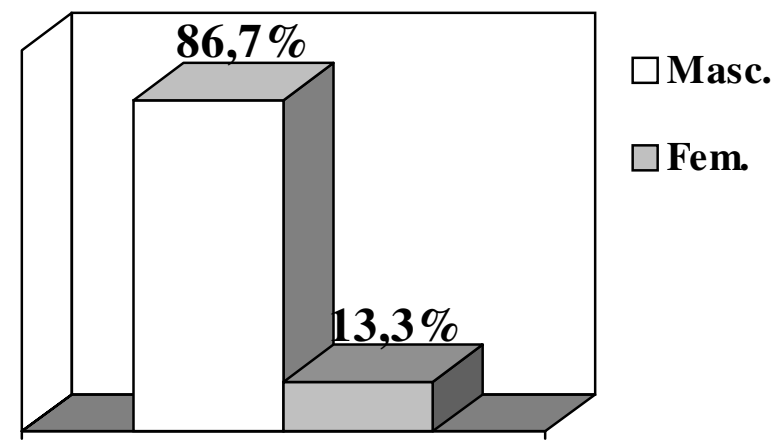


Não houve predominância de fatores de co-morbidade: hipertensão arterial sistêmica estava presente em três pacientes; diabete melito foi evidenciada em apenas um paciente; os demais não apresentavam doenças importantes associadas àquela que motivou o ato cirúrgico e que correspondessem fator de risco para desenvolvimento de mediastinite.

O tempo de permanência em UTI foi de 16 dias (2 a 44 dias), com tempo de permanência em respirador de 25 horas (4 a 90 horas), antes de serem submetidos a qualquer procedimento para tratamento da mediastinite.

Os germes mais freqüentemente isolados foram o S.aureus Oxacilina resistente (63\%) e S.epidermidis (17\%), fazendo com que todos os pacientes fossem tratados com vancomicina associada ou não a outro antibiótico. O tratamento cirúrgico preconizado inicialmente para todos os pacientes foi o debridamento amplo de cavidade mediastinal, com instalação de sistema de irrigação (solução fisiológica aquecida contendo antiséptico) e drenagem mediastinal.

Ressecção total de esterno foi realizada em três (20\%) pacientes, dois dos quais morreram na UTI, sendo que o terceiro paciente foi submetido a três operações plásticas em tórax e, também, a tratamento hiperbárico com oxigênio a $100 \%$.

\section{COMENTÁRIOS}

A baixa incidência encontrada no presente estudo $(1,09 \%)$ está de acordo com taxas semelhantes apontadas na literatura. ZACHARIAS \& HABIB ${ }^{(13)}$, em estudo retrospectivo de quatro anos, em Ohio - EUA, apontam em 2317 operações com esternotomia a ocorrência de $0,91 \%$ de mediastinite, o que se repetiu em estudos de mesmo período realizados em Chicago $(0,92 \%)$ e Nova York $(0,97 \%)$. Índices menores ainda forma observados em estudos de BASKETT et al. (14) que evidenciaram $0,25 \%$ de incidência de mediastinite em 9771 operações realizadas no período de 10 anos, no Canadá. Em Estocolmo, também, há relatos de baixa incidência de mediastinite $(0,43 \%)$, o que não se observa em publicações de SOFER et al. ${ }^{(15)}$, no Hospital Universitário de Tel Aviv - Israel, com 1,7\% de casos observados, bem como no estudo de MISAWA et al. ${ }^{(16)}$, no Japão, que assinalam ocorrência de 2,9\% de pacientes com mediastinite pós-operação cardíaca (722 operações, em 3 anos). No Brasil, por exemplo, recente publicação do Hospital Felício Rocho, em Minas Gerais, mostra incidência de $2,1 \%$ na população estudada (11).

A correlação entre operações de revascularização do miocárdio e o desenvolvimento de mediastinite está bem documentada na literatura, onde se aponta mais de $80 \%$ de casos da infecção como complicação associada à operação (1-4,7,9,12).

A predominância do sexo feminino como um dos fatores de risco para o desenvolvimento de mediastinite não foi observada no presente estudo, devendo-se salientar que a maior parte dos pacientes submetidos à operação de revascularização do miocárdio, em nosso Serviço, são do sexo masculino, razão pela qual estamos trabalhando com uma amostragem populacional viciada ${ }^{(17-19)}$.

Diagnosticar o processo infeccioso antes da alta hospitalar é a forma largamente difundida na literatura como importante para o adequado controle da infecção, uma vez que permite a instituição de medidas terapêuticas precoces, o que reduz significativamente os índices de óbito em função de mediastinite, os quais podem chegar até $40 \%$.

Terapêutica cirúrgica agressiva, com debridamento e instalação de sistema de irrigação, apesar de controversa, ainda é a modalidade de tratamento mais difundida na literatura, mostrando em diversos trabalhos controle e erradicação adequados da doença. Em nosso Serviço, também damos preferência pela aplicação desta técnica de tratamento, razão pela qual, talvez, tenhamse obtido baixos índices de mortalidade decorrentes da mediastinite.

\section{CONCLUSÃO}

A incidência de mediastinite em nosso Serviço foi uma das menores encontradas na literatura especializada. No entanto, não foi possível, no presente estudo, determinarmos os fatores determinantes destes resultados.

Cuidados profiláticos pré e intra-operatórios, bem como a instituição de medidas clínicas e cirúrgicas precoces, podem ter, provavelmente, contribuído para obtenção de índices tão baixos desta complicação infecciosa.

RBCCV 44205-584

Schimin LC, Batista RL, Mendonça FCC - Incidence of mediastinitis at the Hospital de Base do Distrito Federal in six years of study. Rev Bras Cir Cardiovasc 2002; 17(2): 35-38.

ABSTRACT: We review all hospital recorders of the patients that was submitted to cardiac surgery with sternotomy at the Hospital de Base do Distrito Federal in period of six years. Fifteen patients $(1.09 \%)$ developed mediastinitis in this period in a total of 1388 cardiac surgeries between January 1995 and March 2001. Sex, age, type of surgery, respirator time, ICU permanence, use of antibiotics and type of bacteria isolated were some variables analyzed.

DESCRIPTORS: Mediastinitis, surgery. Cardiac surgical procedures, adverse effects. Sternum, surgery. Osteomyelitis, sternum. 


\section{REFERÊNCIAS BIBLIOGRÁFICAS}

1. Fonkalsrud EW - Chest wall abnormalities. In: Glenn WWL, ed. Glenn's thoracic and cardiovascular surgery. 6th ed. vol. 1. Stanford, Connecticut: Appleton \& Lange, 1996. 581-92.

2. Rupp ME - Mediastinitis. In: Mandell R, ed. Mandell: principles and practice of infectious diseases. 5th ed. Churchill: Churchill Livingstone, 2000. 941-9.

3. Lutwick LI; Vaghjimal A; Connolly MW Postcardiac surgery infections. Crit Care Clin 1998; 14: 221-50.

4. Abter El, Lutwick SM, Chapnick EK et al. Mucormycosis of a median sternotomy wound. Cardiovasc Surg 1994; 2: 474-7.

5. Bitkover CY, Gardlund B, Larsson SA, Aberg $B$, Jacobsson $\mathrm{H}$ - Diagnosing sternal wound infections with 99mTc-labeled monoclonal granulocyte antibody scintigraphy. Ann Thorac Surg 1996; 62:1412-7.

6. Demmy TL, Park SB, Liebler GA et al. - Recent experience with major sternal wound complications. Ann Thorac Surg 1990; 49: 458-62.

7. El Oakley RM \& Wright JE: Postoperative mediastinitis: classification and management. Ann Thorac Surg 1996; 61:1030-6.

8. He GW, Ryan WH, Acuff TE et al. - Risk factors for operative mortality and sternal wound infection in bilateral internal mammary artery grafting. J Thorac Cardiovasc Surg 1994; 107:196-202.

9. Hetzer R \& Portner PM - Discussion of infection. Third International Conference on Circulatory Support Devices for Severe Cardiac Failure. Ann Thorac Surg 1996; 61: 372.

10.Ivert T, Lindblom D, Sahni J, Eldh J Management of deep sternal wound infection after cardiac surgery - Hanuman syndrome. Scand J Cardiovasc Surg 1991; 25:111-7.
11. Sampaio DT, Alves JCR, Silva AF et al. Mediastinite em cirurgia cardíaca: tratamento com epíploon. Rev Bras Cir Cardiovasc 2000; 15: 23-31.

12. Parisian Mediastinitis Study Group - Risk factor for deep sternal wound infection after sternotomy: a prospective, multicenter study. J Thorac Cardiovasc Surg 1996; 111:1200-7.

13.Zacharias A \& Habib RH - Factors predisposing to median sternotomy complications: deep vs superficial infection. Chest 1996;110:1173-8.

14. Baskett RJ, MacDougall CE, Ross DB - Is mediastinitis a preventable complication? A 10-year review. Ann Thorac Surg 1999; 67:462-5.

15. Sofer D, Gurevitch J, Shapira I, Paz Y, Matsa M, Kramer A, Mohr R -Sternal wound infections in patients after coronary artery bypass grafting using bilateral skeletonized internal mammary arteries. Ann Surg 1999; 229:585-90.

16. Misawa Y, Fuse K, Hasegawa T.- Infectious mediastinitis after cardiac operations: computed tomographic findings. Ann Thorac Surg 1998; 65: 6224.

17. Prevost LG, Subramainian VA, Rothaus KO, Dineen $\mathrm{P}$ - Comparison of the open and closed methods in the initial treatment of sternal wound infections. J Cardiovasc Surg 1989; 30: 757-63.

18. Acinapura AJ, Godfrey N, Romita M et al. Surgical management of infected median sternotomy: closed irrigation vs muscle flaps. J Cardiovasc Surg 1985, 26: 443-6.

19. Karwande SV, Renlund DG, Olsen SL et al. Mediastinitis in heart transplantation. Ann Thorac Surg 1992; 54:1039-45. 